XVII.

\title{
Rndimentär entwickelte missbildete Ohrmuschel mit Atresie des Gehörganges, Fistulae auriculae congenitae und Hemiatrophia facialis, wahrscheinlich in Folge congenitaler Hypoplasie des paralytischen Nervus facialis. \\ Von
}

Dr. Martin Sugar in Budapest.

(Mit 2 Abbildungen.)

In keinem einzigen Falle unserer Fachliteratur fand ioh die Combination von missbildeter Ohrmuschel, Atresie des Gehörganges mit Hemiatrophia facialis in Folge congenitaler Bypoplasie und Lähmung des $\mathrm{N}$. facialis derselben Seite beschrieben. Aus der Statistik Bezold's (Ueberschau über den gegenwärtigen Stand der Ohrenheilkunde nach den Ergebnissen einer 25jährigen Beobachtung 1895), geht hervor, dass dieser Autor unter einem Gesammtmaterial von 20468 Ohrenkranken nur $11 \mathrm{mal}$ angeborene Atresie des äusseren Gehörganges mit missbildeter Ohrmuschel fand.

Ist damit schon die relative Seltenheit dieser Missbildung zur Genüge erhärtet, so rechtfertigt die bisher nicht beschriebene Combination mit Facialislähmung resp. Hypoplasie des Gesichtsnerven in einem Falle meiner Beobachtung die Pablication des nachstehenden hochinteressanten Krankheitsbildes, das sowoll für den Otiater, als für den Nervenarzt von Bedeutung erscheint.

Der in Budapest wohnhafte, 43 Jahre alte Rechnungsrevisor des königl. ungar. Finanzministeriums $\mathrm{J}$. Chr. suchte mich in meiner Privatordination mit folgenden Klagen auf. Vor einigen Tagen erkrankte er nach einer heftigen Erkältung an unertrăglichem Ohrsebmerz und Ohrensausen im linken Ohre. Ein in Ofen wohnhafter praktischer Arzt verordnete ihm ein Gehöröl, worauf das Sausen stetig zunahm. Da das Letztere ibm, dem mit Zahlen angestrengt geistig arbeitenden Manne, selbst die Nachtruhe raubt und ihn zur Verzweiflung treibt, so dass er sich mit Selbstmordideen trage, er überdies in der letzteren Zeit Zwangsvorstellungen habe (z. B. zweimal zwei sei nicht vier u-s.w.), suchte er meine specialärztliche Hilfe auf. $\mathrm{Pa}$ tient bemerkt noch, dass er das Sausen deshalb so intensi zu bören glanbe, weil es beim anderen, also rechten, seit seiner Geburt verkruppelten Ohre nicht aus dem Kopfe herausdringen könne. Ohrenkrank soll er alusserdem niemals gewesen sein. Schon bei der äusseren Besichtigung fiel mir die Asymmetrie des Kopf- und Gesichtsschădels auf. Der Kopfschädel ist eigen- 
thümlich verbildet, indem er transversal entsprechend der Kronennaht eine tiefe Einsattlung zeigt. Wir haben es mit einer ausgeprägten sogenannten Klinocephalie, mit einem Sattelschädel zu thun, der bekanntlich dadurch entsteht, dass die Nabtverbindung zwischen dem vorderen unteren Winkel des Scheitelbeins und dem angrenzenden Theile des Keilbeins frühzeitig obliterirt. Ob auf die Entstehung dieses verbildeten Schädels nicht anch der seit der Geburt einseitig fehlende Zug der rechtsseitigen Kaumusculatur, ein neben der frühzeitigen Synostose der Nähte ebenfalls wiebtiger Factor der Schädelconformation, mitgewirkt hat, will ich später erörtern

Das Gesicht des Patienten ist ausgesprochen asymmetrisch. Dis ganze rechte Gesichtshälfte ist schmäler, kleiner und in der Entwicklung auffallend zurückgeblieben. Haut, Bindegewebe, Fettgewebe, aber auch Muskeln dieser Gesichtshälfte sind deutlich atrophisch. Das Kinn ist förmlich in zwei Hälften gespalten, indem die rechte Kinnspitze bedentend höher steht. Die Stirne dieser Seite zeigt keine transversalen, sondern nur sagittale Furchen und Falten; sie ist glänzend, auch steht sie höher und bleibt vel der Aufforderung, die Stirn zu runzeln, zurück. Die Augenbrauen stehen etwas tiefer, als auf der gesunden linken Gesichtshălfte.

Das obere Augenlid erscheint auf der rechten Seite schmäler, als aut der linken Gesichtshälfte. Bei begehrtem Lidschlag durch Augenschluss, wird die Lidspalte dieser Seite nicht ganz geschlossen. Die Nasenspitze weicht gegen die linke Seite ein wenig schief ab. Die Mundspalte ist schief verzogen und bleibt beim Pfeifen offen. Keine Sensibilitätsstörungen, keine Geschmacksstōrungen; Zungen- und Kaubewegung, Thränen-Speichelsecretion ungestört. Die Zunge ist jedoch auf der rechten Hälfte runzlich, schmäler, weniger convex, als auf der linken Hältte, somit deutlich atrophisch. Bei geöffnetem Munde finden wir deutliches Tieferstehen der Gaumenbögen der rechten Seite, doch keinen Schiefstand der Uvula. Schon dieser genauer präcisirte Befund weist auf eine rechtsseitige Gesichtsnervenlähmung mit Atrophie der Gesichtsmuskeln und der Gesichtshaut hin, welch letztere für die lange Dauer der Facialislähmung spräche.

Bei Untersuchung des Ohres, dieser zunächst unsere Aufmerksamkeit orregenden Seite, finden wir die Ohrmuschel nur rudimentär angedeutet und eine rollständige Atresie des äusseren Gehörganges. Wir finden eigentlich nur zwei Wülste durch eine Furche getrennt; die Haut über derselben ist normal. Bei Betastung erscheint der untere Wulst weich, so dass schon die äusseren Kennzeichen dem lobulus entsprechen. Der obere Wulst imponirt als Knorpelgebilde und scheint dem missbildeten Antitragus oder der verkrüppelten Helix zu entsprechen. Tragus, Concha, Antihelix, Fossa scaphoidea, Meatus, Spina helixis, Crura furcata, Fossa intercruralis fehlen.

Am Lobulus zwei kleine, fast in einer Horizontalen gelegene, nicht secernirende Fistelöffnungen, die nur im Anfange mit einer Borstensonde passirbar, blind endigen und die wir als echte Auricularfisteln bezeichnen können.

An der Stelle des atresirten äusseren Gehörganges ein wallartig begrenzter Knochenring, der dem Annulus tympanicus entspricht. Der Warzenfortsatz dieser Seite ist minder entwickelt, als auf der linken Seite.

Das linke Ohr, weswegen der Patient meine Hilfe aufsuchte, zeigt die Verănderungen eines subacuten Mittelobrkatarrhs. Bei Stimmgabeluntersuchung wird Weber nach links lateralisirt, Rinne links positiv, rechts negativ. Tiefe Stimmgabel rechts nnr ad concham rudimentariam, doch undeutlich, rom Schlăfenbein aus deutlicb. Uhr rechts nicht einmal ad concham, ja selbst nicht einmal vom Schläfenbein aus percipirt. Hobe Stimmgabel $\left(\mathrm{c}^{2}\right)$ wird vom Scheitel rechts entschieden besser vernommen. Die Flüsterstimme wird rechts vom missbildeten Obre in der Distanz von $11 / 2 \mathbf{m}$ gehört; in diesem Obre seit Langem ein an feines Vogelzwitschern erinnern. des Geräusch, links im acut erkrankten Ohre Locomotivpfeifen, Läuten, dröhnendes Hämmern.

Behandlung des linken Ohres: Carbolglycerin, Politzer'sche Luftdouche, später Kampherätherinjectionen, Delstanche, Pneumomassage, elektrischer Strom, intern Bromnatrium. 
Sehr langsame Besserung, die sich alsbald wohl durch Schwinden der Schmerzen kundgiebt, doch das sausen besteht in mässigem Grade noch immer und wird zeitweise in das missbildete Ohr verlegt. Bei Anwendung der Luftdouche nur einseitiges Lindringen des Luftstromes in das linke Ohr; der Katheter ist nicht $\mathrm{jm}$ Stande den maximal verengten Nasengang der rechten Nasenhälfte zu passiren; bei der Rhinoscopia posterior Fehlen des Tubenwulstes; es scheint somit Atresie der Eustacbi'schen Ohrtrompete zu bestehen.

Wenn wir das eben beschriebene seltene Krankheitsbild analysiren, wenden wir uns mit Recht dem verbildeten rechten Ohre zu. Auffallend ist die balbseitige Gesichtslähmung, die Hemiatrophie und Asymmetrie der Gesichtshälfte, die rudimentär entwickelte Ohrmuschel und die Atresie des Gehörganges dieser Seite.

Was zunächst die Asymmetrie betrifft, finden wir fur dieselbe in der Facialisparalyse und der durch dieselbe verursachten Gesichtsmuskelatrophie genügende Erklärung. Bei der Entstehung der Gesichtsasymmetrie concommittiren aber auch andere Momente. Dieselbe ist nämlich auch ohne bestehende Facialislähmung zu erklären.

Lucae erklärt die bei Atresia auris congen, vorkommende Gesichtsasymmetrie aus der mangelhaften Entwicklung der Gesichtsknochen dieser Seite (S. Anatom. physiolog. Beiträge zur Obrenheilkunde, Virchow's Arehiv XXX, S. 63).

Nach Za ufal verdankt die Gesichtsassymetrie dem Fehlen der Pars tympanica des Schläfebeins ihre Genese. "Durch das einseitige Fehlen der Pars tympanica des Schläfebeins rückt der Proc. condyl. des Unterkiefers weiter nach rückwärts, als auf der normalen Seite, so dass er unmittelbar vor dem Proc. mastoid. zu fühlen ist. Durch diese Rückwärtslagerung eines Unterkieferastes kommt die Asymmetrie der betreffenden Gesichtshälfte allmählich zu Stande und zwar oft mit Eintritt des Kaugeschäftes, durch welches die Stellung des Unterkiefers sich mehr regulirt." (Prager med. Wochensehrift 1876, S. 859). Durch diese soeben erwäbnten geistreichen und auch plausiblen Hypothesen kann ich aber nur die Gesichtsasymmetrie, keineswegs die Lähmungen im Bereiche des Gesichtsnerven und den halbseitigen Gesichtsschwund erklären.

Wie oben erwähnt, können wix dureh Atrophie der Kaumuskeln ganz gut auch die Verbildung der Schädelkapsel in Folge einseitig wirkenden Zuges erkiären.

Diese Atrophie kann aber auch dadurch zu Stande kommen, dass in Folge congenitaler oder frühzeitiger Lähmung, resp. 
Atrophie der Zweige des Pes anterinus major Nervi facialis (Rami zygomatici, boccales), die bekanntlich mit dem motorischen Nerven des Trigeminus anastomosirend, die Kaumuskeln versorgen, die letzteren einem früheitigen Schwund anheimfallen. Aus den experimentellen Untersuchungen von Bracke und Brown-Séquard wissen wir, dass eine Durchsehneidung des Facialis bei jungen Thieren die entsprechenden Muskeln atrophisch macht, wodurch auch die Gesichtsknochen im Wachsthum zurückbleiben, sie bleiben kleiner und es wachsen daher die Gesichtsknoohen der intacten Seite schliesslioh über die Mittellinie hinans, gegen die afficirte Seite gewendet. (S. Landois Physiologie. I. Aufl. S. 681.) Aebnliches finden wir bei der Hemiatrophia facialis progressiva, bei jener räthselhaften, stets acquirirten Krankheit, die man als Trophoneurose des Gesichtsnerven, oder nach der neuesten Anschauung auf Grund der Sectionsergebnisse Mendel's, als Folgeerscheinung der Atroplie der linken absteigenden Trigeminuswurzel und interstitielle Neuritis proliferans dieses Nervenzweiges auffasst. (S. EbsteinSchwalbe's Handbuch IV. Band. S. 645 „Erkrankung der peripher. Nerven" von Eulenburg.)

Ein auffallendes Symptom finden wir, das der Bemiatrophia facialis zukäme; ich meine die gewiss auffallende halbseitige Zungenatrophie in unserem Falle, die wir nur mit der einseitigen Hypoplasie des knöcheruen und musculösen Schädelskelettes erklären können. Hierbei sind wir wieder bei der besonders interessanten Lähmung des $\mathrm{N}$. facialis im oben beschriebenen Falle angelangt. Ich glaube es aussprechen zu können, dass der N. facialis congenital gelähmt, oder mindestens hypoplastisch entwickelt ist. Anton beschrieb einen Fall von Atresie des Gehörganges mit missbildeter Ohrmuschel, combinirt mit totaler Lippenkiefergaumenspalte, allerdings bei einem 19 Tage alten Nengeborenen, und fand, anatomisch, eine Hypoplasie der rechten Pyramide, sowohl in deren Längs- als Diekendurchmesser, obwohl er den Nervus facialis und actsticus normal fand (Prager med. Wochenschrift 1897. S. 235.)

Das Vorkommen von Defecten am knöchernen Schädelskelette und an den Weichtheilen des Gesichtes bei rudimentärer Entwicklung der Ohrmuschel und Atresie des Gehörganges wird insbesondere ron Moldenhauer (Schwartze's Handbuch I, Missbildungen) betont. Bekannt sind Hasenscharten, Gaumenspalten, Wolfsrachen, Ohr- und Halskiemenfisteln. 
Aus den von Jö̈l zusammengestellten Sectionsbefunden (Ueber Atresia aurieul. cong. Inaug.-Dissert. 1888), Steinbrügge (Deutsche otol. Gesellschaft in Hamburg 1899 20. Mai, Monatsschrift f. Ohrenheilk. 1899) und Ruedi (Z. f. 0. 34.4 S. 334, 1899) geht hervor, dass die Paukenhöhle selbst in den meisten Fällen durch Knochenmassen eingeengt, oder von solchen gänzlich ausgefullt war, wie dies auch neuerdings Blan in seinem mit grossen Fleisse zusammengestellten, vollkommenen Beriehte über die neueren Leistungen in der Obrenheilkunde (Schmidt's Jabrbileher Bd. CCLXXV "Aeusseres Ohr") hervorhebt. Ioh glaube kaum fehlzugehen, wenn ich annehme, dass im gegenwärtigen Falle der N. facialis schon seit Geburt des Patienten, vielleicht gar intrauterin, durch Knochenbalken entweder im Fallopischen Canale, oder in der Gegend des For. stylo-mastoideum gedrückt wurde und atrophisirte. Möglich wäre auch bei einer vorhandenen Hypoplasie des Schläfebeins eine Einengung seines Verlaufes innerhalb des Felsenbeines und agenetische, hypoplastische Entwicklung desselben.

Zur präcisen Localisirung der Stelle der Facialisaffection könnte man wohl die Ausdehnung der Gesichtsnervenlähmung, resp. das Ergriffensein der einzelnen Gesichtszweige des $\mathbf{N}$. facialis heranziehen, doch hierbei finden wir eine neue Schwierigkeit in der Deutung unseres Krankheitsbildes.

Sind Lähmungursachen innerhalb des Canalis Fallopiae dicht iber dem N. petrosus superficialis major, oder am Ganglion geniculatum selbst gelegen, so kommen zu den Lähmungserscheinungen an den Gesichtsohrmuskeln und am M. occipitalis, Geschmacks-Secretions- und Gehörstörungen, noch Lähmung des Gaumens hinzu. Die activen Lähmungserseheinungen an den Ohrmuskeln können wir in unserem Falle nicht constatiren, da ja das $\mathrm{Ohr}$ der bezüglichen Seite rudimentär entwickelt ist, den Occipitalmuskel können die wenigsten Menschen activ bewegen und die entscheidende Gaumenlähmung ist wobl in ausgesprochener Weise vorhanden, doch ist dieses Symptom speciell bei Atresie des Gehörganges zweideutig. Politzer hebt hervor (Lehrbueh 1901), dass bei vorhandener Atresie stets die Bewegungen des Gaumensegels während der Phonation zu prüfen sind. Findet man eine gleichmässige Beweglichkeit der Gaumenklappe, so kann mit Wahrscheinlichkeit auf eine normale Entwicklnng der Ohrtrompete und des Mittelohres geschlossen werden. "Zeigt die Gaumenhälfte der missbildeten 
Seite eine geringere Beweglichkeit, so ist die Annahme einer Defectbildung der Trommelhöhle und der Ohrtrompete gerechtfertigt, weil die mangelhafte Entwicklnng des Mittelohres mit einer solchen der Gaumenmusculatur verbunden ist."

Den Defect der Ohrtrompete habe ich in meinem Falle constatirt und wir sehen daher, dass die mangelnde Bewegliehkeit der Gaumenhälfte nicht Ausdruek der Facialislähmung sein muss. Ja auf Grund nenerer literariseher Angaben kann ich

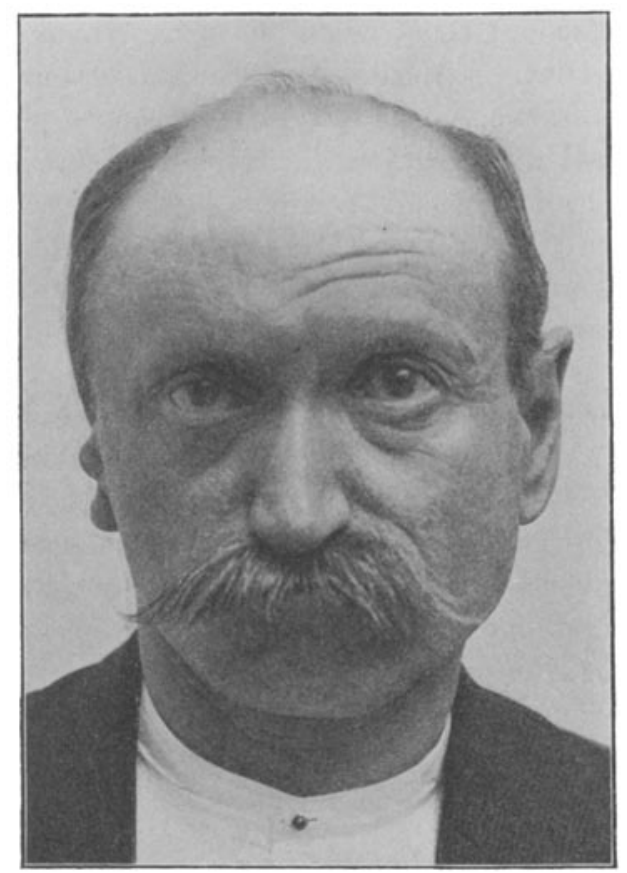

Fig. 1.

mit meinen Schlüssen noch weiter gehen. Ein gewiss isachkundiger Neurologe, wie Eul enburg, giebt an, dass der Facialis an der Innervation des Gaumensegels keinen directen Antheil hat, denn der motorisehe Charakter des N. vidianus und seines $\mathrm{N}$. petrosus superficialis major ist nicht mehr so nnbestritten, wie fruher, vielmehr ist dieser Nerv wahrscheinlich ganz und gar oder tiberwiegend sensibler Art. (Ebstein-Sehwalbe, S. 655, IV. Band.)

Am wahrscheinlichsten ist noch die rejne Prosoplegie durch 
Ursachen möglichst peripher und ausserhalb des For. stylomastoidenm, da Patient seit seiner Geburt ein schiefes Antlitz hat und Geschmacks-Secretionsstörungen fehlen.

Dies war mein Raisonnement, als ich mich an unseren Neurologen Prof. Ernst Jendrassik mit der Bitte wandte, die elektrische Reizbarkeit der hemiatrophischen Gesichtshälfte auf das Genaueste zu prüfen. Den minutiös genauen Befund Jendrassik's will ich wörtlich anfübren: „Elektrische Erregbarkeit normal, doch tritt bei derselben Stromstärke auf der reohten (atrophischen) Gesichtshälfte (Stirn, Wange) rascher eine Reaction ein. Die elektrische Erregbarkeit ist im Uebrigen sowohl dem faradischen, wie dem galvanischen Strom gegenüber, und zwar sowohl die Reaction der Muskel - als Nervenpunkte, als normal zu bezeichnen. Zur Reizung des rechtsseitigen Musculus quadratus menti ist ein um vieles stärkerer Strom nothwendig, doch auch hier fehlt die, Entartungsreaction'. M. retrahens auriculae und $M$. occipitalis reagiren prompt."

Die stärkere Erregbarkeit der oberen Zweige des N. facialis bezieht Jendrassik darauf, dass der Facialis nicht von Fettpolster gedeckt, somit freier liegt. Denn anch das Unterhautzell- and Fettgewebe participirt an der Hemiatrophie des Gesichtes. Unleugbar aber bleibt die ausgesprochen torpide Reaction des M. quadratus menti. Jendrassik sehliesst von seinem Standpunkte als Nenrologe, nicht vollkommen die Hypoplasie des N. facialis aus, neigt aber zur Auffassung, dass diese nur Theilerscheinung der Agenesie des Gesichtsschädels sei. Der besonderen Freundlichkeit Jendrassik's habe ich anch die beigelegten photographischen Aufnahmen zu danken, die er persönlich in seinem mustergültig eingerichteten Institute, in der Nervenklinik der königl. Universität zu Budapest, verfertigte.

Es eribrigt mir selber noch, auf die Frage des Nähern einzugehen, ob das Fehlen der sogenannten Entartungsreaction die Facialisparese- oder Paralyse aussehliesst? Keineswegs! Denn eine Fachautorität, wie Eulenburg (l. c.), spricht sich über das Ergebniss der elektrischen Reizbarkeit wie folgt aus.

"Bei den ganz leichten Formen der Facialislähmung sind irgendwie in's Gewicht fallende Anomalien des elektrischen Verhaltens an Nerv und Muskel taberhaupt nicht zu erkennen. Bei den sogenannten "Mittelformen", kommt es nach einer kurzen initialen Steigerung der Nervenerregbarkeit gewöhnlich schon 
vom Ende der zweiten Woche ab zu einem Absinken der faradischen und galvanischen Nervenreizbarkeit und etwas später, auch zur Abnahme der faradischen Mnskelreizbarkeit, jedoch nicht bis zu deren völligem Verlorengehen, während gleichzeitig die leichteren Formen der EaR. - erhöbte galvanisehe Erregbarkeit, träger Zuckungsmodus, Ueberwiegen der Anodenschliessungsreaction gegentiber der Kathodenschliessung - sich an den Muskeln entwickeln. Die im engeren Sinne "schweren"

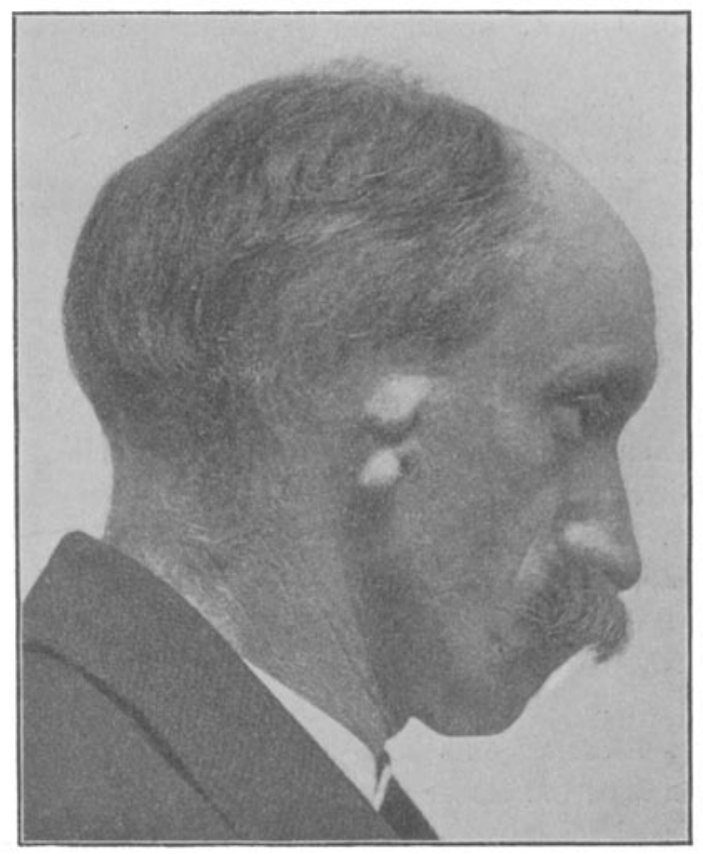

Fig. 2.

Formen charakterisiren sich durch gänzlichen Verlust der Nervenreizbarkeit und faradischen Muskelreizbarkeit and durch die höheren Grade der galvanischen Entartungsreaction - Absinken der galvanischen Muskelreizbarkeit auch für Anodenschliessung, alleiniges Zurückbleiben von An. S. Z. bei starken Strömen und schliesslich totale Unerregbarkeit der Muskeln. Es ist übrigens nicht immer ganz leicht, zu entscheiden, ob die Nervenreizbarkeit (und die faradisehe Muskelreizbarkeit) völlig aufgehoben, oder nur mehr oder weniger stark herabgesetzt ist, da man in manchen Fällen wegen der oft beträchtlichen Empfindlichkeit 
für elektrische Ströme - bei Galvanisation auch aus anderen Rücksichten - über ein gewisses Maass der Stromintensität nicht herausgehen darf. Man muss diese Frage daher öfters unentschieden lassen."

Anlangend die Genese der Facialisparese- resp. Hypoplasie, mag nnerörtert bleiben, ob im Sinne des citirten Joël eine intrauterine luetische Infection die Hand im Spiele hat, denn richtig bemerkt Anton (l.c.), dass die Combination mit sonstigen Missbildungen im Bereiche der 1. Kiemenspalte, in der Gegend des äusseren Ohres, gegen die entzündliche Genese Joël's spricht.

Die Verbildung des äusseren Ohres muss mit Entwicklungsstörungen im Bereiche des 1. Kiemenbogens in Zusammenhang: gebracht werden. Aeusseres und mittleres Obr gehen bekanntlich mit Ausnahme des Steigbügels ans dem 1. Kiemenbogen hervor, während das Labyrinth dem Ektoderme seine Entstehung verdankt. Dahingestellt mag anch bleiben, welcher Natur diese Entwicklungsstörungen sind, die chronologisch wohl vor dem Ende des 2. Monates statthaben, da nach Hertwig am Schlusse des zweiten embryonalen Monates alle wesentlichen Theile des äusseren Ohres leicht erkennbar sind. $\mathrm{Ob}$ amniotische Verwachsungen, deren mechanischen Einfluss Alexander und Moskoviez (Archiv f. Ohrenh. 19110. 1. 2. S. 97) studirten, oder Umschlingungen der Nabelschnur, die nach Moldenhauer (Schwartze, Grosses Bandbuch I, Missbildungen) von den Eltern als Grund angegeben werden, oder Vererbung eine Rolle spielen, ist schwer zu entscheiden.

Die von Molden ba er und Joux herruhrende Behauptung (eitirt in Henke, Monatssehrift f. 0. 1899. 33. 2 S. 55), dass sich keine Unregelmässigkeit des väterlichen Körpers leichter auf die Kinder vererbe, als Ohrmuscheldifformitäten, erwies sich auch in unserem Falle als unzutreffend, da in der Familie des Patienten keine Obrmuscheldifformitäten vorkommen. Auffallend war aber, dass die Missbildung, wie gewöhnlich, auch hier das rechte Ohr ergriff.

Wenn die Entstehung der Fistula auris congenita vielfach Gegenstand von Controversen bildete und speciell in Folge von Untersuchungen von Urbantsehitseh der treffendere Ausdruck "Fistula fissurae bronchialis primae congenita" vorgeschlagen wurde, so kann nach der Arbeit Grunert's kein Zweifel bestehen, dass diese Fistula auriculae congenita aus- 
drücklich auf Störungen in den secundären Entwicklungsvorgängen des äusseren Ohres zurïckzuführen ist, was Grunert durch die histologische Untersuchung des Fistelcanales erwies (in diesem Archiv, Bd. XLV, 1 und 2, S. 14).

Es frägt sich nun, wie sieht das innere $\mathrm{Ohr}$ in unserem Falle aus?

Ich habe bereits hervorgehoben, dass der Patient Flüsterstimme in einer Distanz von $1 \frac{1}{1} 2 \mathrm{~m}$ hört. Dieser Befund, nebst dem Ergebnisse der Stimmgabelprüfung, spricht dafür, dass das Labyrinth vorhanden sei. Hierfür spricht sicherlich auch die stärkere Perception der Stimmgabel $\mathbf{c}^{2}$ vom Scheitel aus auf dem missbildeten Ohre, was Politzer (l. c.) als verlässliches Zeichen hervorhebt. $\mathrm{Blau}$ erwähnt in seinem oben citirten Berichte, dass das innere $\mathrm{Ohr}$ in den meisten einschlägigen Fällen normal war. Aus der Literatur ist der Fall Steinbrüg ge's bekannt (l. c.), dessen Kranker trotz beiderseitigem Verschlusse, dem gewöhnlichen Unterricht zu folgen vermochte.

$\mathrm{Da}$ in den zur Obduction gelangten Fällen stets tiefe Veränderungen vorhanden waren, so Fehlen von Gehörgang und Trommelfell, knöcherne Verengung, oder Fehlen der Pankenhöhle, Abweichungen der Gehörknöchelchen, Defect der Tuba, ist ein selbst event. gelingender operativer Versuch zur Wiederherstellung des fehlenden Gehörganges in Bezug auf Verbesserung des Gehörs nutzlos.

Trotzdem soll nach Angabe des Patienten in der obirurgischen Universitätsklinik zu Klausenburg bereits vor Jahren eine Eröffnung des Gehörganges beabsichtigt worden sein, ein nach dem Entwickelten gewiss eitles Beginnen. Nur aus kosmetisehen Gründen, wenn wir die beschriebene Missbildung des äusseren Ohres etwa bei einem jungen Mädchen fänden, könnten wir an die Abtragung der rudimentären Ohrmuschel und an arteficiellen Ersatz durch eine Prothese denken. :Diesbeziuglich empfehlen sich die vom Collegen Dr. Henning in Wien bereiteten künstlichen Ohrmoulagen, die sowohl betreffs vollendeter Form, als Dauerhaftigkeit einen Fortschritt bedeuten. 